\title{
Venous Ulcer: Review Article
}

\author{
Shashi Prakash, Satyendra Kumar Tiwary, Manjaree Mishra, Ajay Kumar Khanna* \\ Department of General Surgery, Institute of Medical Sciences, Banaras Hindu University, Varanasi, India \\ Email: sprakashsurgery@gmail.com,drsktiwary1@gmail.com,drmanjareemd@gmail.com, *akhannabhu@gmail.com
}

Received December 4, 2012; revised January 2, 2013; accepted January 11, 2013

\begin{abstract}
Venous ulceration is the most severe and debilitating outcome of chronic venous insufficiency in the lower limbs and accounts for 80 percent of lower extremity ulcerations. The morbidity caused by them has a serious impact on the quality of life. Sustained venous hypertension, caused by venous insufficiency leads to venous ulceration. The diagnosis is mainly clinical but needs to be differentiated from other causes of lower limb ulcers. Doppler ultrasound is the diagnostic investigation. Treatment options for venous ulcers include conservative management, mechanical treatment, medications, and surgical options. The goals of treatment are to reduce edema, improve ulcer healing, and prevent recurrence. The achievement of good long term results depends on continuous care, ulcer care clinics, home health nursing and regular evaluation by the doctor. They have a crucial role to play for the amelioration of this common and morbid condition.
\end{abstract}

Keywords: Venous Ulcer; Chronic Venous Insufficiency

\section{Introduction}

Venous ulceration (stasis ulcer) is the most severe and debilitating outcome of chronic venous insufficiency in the lower limbs and accounts for 80 percent of lower extremity ulcerations [1]. Various other causes for lower extremity ulcerations could be arterial insufficiency, prolonged pressure, diabetic neuropathy and systemic illness such as rheumatoid arthritis, vasculitis, osteomyelitis, and skin malignancy [2]. In the United States overall prevalence of venous ulcers is approximately 1 percent [1]. Venous ulcers are more prevalent in women and older persons and the risk factors primarily include older age, obesity, previous leg injuries, deep venous thrombosis and phlebitis [3-7]. They are often recurrent and may sometimes persist from weeks to years, giving rise to the complications in form of cellulitis, osteomyelitis and at times malignant change [3,8-10]. In spite of low overall prevalence, the refractory nature of these ulcers causes an increased risk of morbidity, mortality and a significant impact on quality of life [11,12]. The financial burden of venous ulcers is estimated to be $\$ 2$ billion per year in the United States [13,14].

\section{Aim and Objective}

The aim of this review article is to elaborate the various aspects related with pathophysiology, diagnosis and treatment of venous ulcers.

\footnotetext{
${ }^{*}$ Corresponding author.
}

\subsection{Pathophysiology}

The circulatory system of the body is a complex network of blood vessels, if they become diseased or damaged then skin breakdown can occur, or an existing wound could fail to heal. Veins usually carry deoxygenated blood towards the heart and are often closer to the skin than arteries. Most veins have one-way flaps or valves that prevent backflow of blood and pooling in the lower legs. These valves may become diseased or damaged, hence incompetent, causing blood to flow back. This leads to an increase in pressure within the deep venous system causing the vein walls to stretch, opening up the valves and allowing even more blood to fill the veins. Veins are classified as superficial and deep. Superficial veins are close to the body surface having no corresponding arteries. The long saphenous vein is the most important superficial vein in the lower limb, running from the dorsal vein at the big toe to join the femoral vein at the sapheno-femoral junction at the top of the thigh. Deep veins are found much deeper within the leg and all have corresponding arteries, with similar names, running alongside them.

Examples are the perineal and femoral veins. It is within the deep veins where blood clots form, causing a deep vein thrombosis (DVT), which is one of the main causes of venous hypertension. Perforator veins connect the deep and superficial venous systems and have one way valves. 


\subsection{Venous Leg Ulceration}

Sustained venous hypertension, caused by venous insufficiency leads to venous ulceration. Incompetence of superficial veins and/or of perforators (because of direct injury, congenital abnormality or superficial inflammation) causes $40 \%-50 \%$ of venous leg ulcers. Deep venous system in these patients, function as normal [15].

Veins can also be damaged by surgery, trauma or DVT, causing a backflow of blood in the venous system at the point of damage. Other causative factors include multiple pregnancies, obesity, congenital vein abnormalities and varicose veins.

To compensate for the increased blood in them because of pooling, wall of the veins gets stretched up but this causes venous hypertension in turn. Hence within the circulatory system, unequal pressure develops between the arteries and the veins and which if left untreated, may lead to chronic venous hypertension. The venous hypertension causes red blood cells to leak into the extra vascular space. Haemosiderin is released from the red blood cells and breaks down.

This fluid then leaks out of the stretched veins into the tissues, depositing a brown/red pigment in the gaiter area of the leg. Venous ulceration occurs in the gaiter area in $95 \%$ of cases especially, around the malleolar region [15]. Waste products from blood proteins and fibrinogen also leak into the tissues, which can irritate the skin causing venous eczema. Patients with venous disease also remain at an increased risk of developing allergic reactions to certain products known as allergens because of this process. Leg ulcer allergens can be found in a wide range of topical treatments such as emollients, medicaments, dressings, bandages and hosiery [16].

Failure of calf muscle pump is another factor that influences the development of venous leg ulcers. The calf muscle, through contraction and relaxation, aids in the flow of blood back to the heart through the veins. In the flexed ankle, the shape of the calf muscle changes, becoming wider and flatter, exerting pressure on the veins [17]. Failure of this mechanism causes stasis of blood and increased venous pressure. Failure of calf muscle pump may arise because of immobility, paralysis and sleeping in chair for prolonged period with legs dependent and fixed ankle joint.

Venous hypertension leads to certain skin changes which can be helpful for supporting the etiology of the leg ulcer. Increased pressure in the capillaries causes dilation and leakage of fluid into the subcutaneous cells and into the interstitial spaces, which causes edema. Gaseous exchange is slower within the microcirculation and there is reduced tissue oxygenation.

The tiny veins on the medial aspect of the ankle dilate due to the increase in pressure. This is known as ankle flare and also causes atrophy of the veins, which are prone to damage, especially around the lower ankle area.

Chronic venous hypertension causes tissue fibrosis and a woody appearance. The shape of the lower leg can change into what is described as a "champagne bottle" appearance. Lipodermatosclerosis is a term used to describe the combination of changes in the lower leg from venous hypertension. As a result of venous congestion and the loss of intercellular fluid balance, the skin can become very dry and flaky. The build-up of hyperkeratotic skin is also an indicator of high venous pressure. In venous disease, ulcers are usually located in the gaiter area between the ankle and the calf, often on the medial aspect of the leg.

\subsection{Clinical Presentation and Diagnosis}

It is a critical part of management to determine the etiology of venous ulcer to plan for proper management of venous ulcers. There are characteristic differences in clinical presentation and physical examination findings which can help to differentiate venous ulcers from other lower extremity ulcers [2]. The diagnosis of venous ulcers is generally clinical; however, investigations such as ankle-brachial index, color duplex ultrasonography, plethysmography, and venography may be helpful in doubtful cases [18-21].

\subsection{Clinical Presentation}

Venous stasis commonly presents as heaviness, dull ache or pain in the lower extremities and swelling that subsides with elevation. Skin discoloration, eczematous changes of the surrounding skin, and varicose veins may be seen [2]. Venous ulcers often occur over bony prominences, particularly the gaiter area and recurrence of an ulcer in the same area is highly suggestive of venous ulcer.

On physical examination, venous ulcers are commonly irregular shape and shallow ulcers. Granulation tissue and fibrin are often present in the ulcer base. Other findings include lower extremity varicosities; edema; venous dermatitis associated with hyperpigmentation and hemosiderosis or hemoglobin deposition in the skin; and lipodermatosclerosis associated with thickening and fibrosis of normal adipose tissue under skin. A clinical severity score based on the CEAP (clinical, etiology, anatomy, and pathophysiology) classification system can guide the assessment of chronic venous disorders. The highest CEAP severity score is applied to patients with ulcers that are active, chronic (greater than three months duration, and especially greater than 12 months duration), and large (larger than $6 \mathrm{~cm}$ in diameter) [22,23]. Poor prognostic factors for venous ulcers include large size and prolonged duration $[24,25]$. 


\subsection{Investigations}

Hand held Doppler is very useful and sensitive device to assess blood flow in the legs and to measure the blood pressure at the ankle, to calculate ankle brachial pressure index (ABPI). Usually the flow of blood in the arms and legs is the same and the index is around 1.0. If there is impairment of the circulation in the lower limbs then the index will be reduced to less than 1.0. The quality of the Doppler signal has also got its important. A biphasic or triphasic signal indicates good arterial flow even if the ABI is difficult to measure or pulses cannot be felt. It does need experience to be able to evaluate the Doppler accurately. Hand Held Doppler can also be used in the clinic to assess the veins, but the assessment is not as accurate as that in the arteries. Assessment of the veins using Doppler ultrasound supplies detailed information about the anatomy of the system of veins and the direction of blood flow in the veins.

Unusual appearing ulcer or if the ulcer persistently fails to heal, a tissue biopsy is deemed important. It rules out underlying malignancy and may also help with other diagnoses in certain situations.

\subsection{Continuous Wave Doppler Ultrasonography}

Doppler ultrasound scanning has become integral part of the physical examination in clinical vascular practice. It is very useful in assessing arterial and venous patency and to ascertain the presence of venous reflux. Venous reflux is detected keeping patient in upright while the following sites are investigated: the greater saphenous vein at the junction and along its course and the lesser saphenous vein in the popliteal fossa and distally. Perforating veins should be sought. The patency and competency of the femoral vein in the groin, the popliteal vein, the posterior tibial vein behind the medial malleolus, and the anterior tibial vein can also be ascertained. Spontaneous flow cannot be detected with an $8.0-10.0 \mathrm{MHz}$ probe when the patient is upright. Instead, augmented flow in the various venous segments can be elicited by manual compression of the sole of the foot or calf. Releasing the compression will elicit reverse flow through incompetent valves. Venous reflux can also be elicited by a deep inspiration, a Valsalva maneuver, or proximal compression.

Proximal obstruction to flow can be detected in a supine patient by observing the venous flow during spontaneous breathing. Continuous flow unaffected by movement of the abdominal wall may be noted when the affected leg is compared with the other leg. This may give useful information about the degree of proximal obstruction.

\subsection{Duplex Doppler Ultrasonography}

It allows for both anatomical and functional assessment of the venous system and can also measure flow velocity, flow rate, and the diameter of the veins. It can also visualize deep veins to reveal occlusion, recanalised lumen and irregularities in vein walls. Incompetent perforating veins can also be visualized. Real time color duplex scanning makes orientation of venous flow much easier and provides information about reflux in specific veins. Phlebography is reserved for special situations.

\subsection{Treatment}

Treatment options for venous ulcers include conservative management, mechanical treatment, medications, and surgical options [1,2,7,10,22,25-47]. The goals of treatment in venous ulcer are to reduce edema, improve ulcer healing, and prevent recurrence. Although numerous treatment methods are available, they have variable effecttiveness and limited data to support their use.

\section{Conservative Management}

\subsection{Compression Therapy}

Compression therapy has been recognized as the standard of care for venous ulcers and chronic venous insufficiency $[26,48]$. A recent Cochrane review found that venous ulcers heal more quickly with compression therapy than without it [48]. Compression can be provided by inelastic, elastic, and intermittent pneumatic compression. Compression therapy has been shown to reduce edema and pain, improve venous reflux and enhance healing of ulcers [26]. Success rates may range from 30 to 60 percent at 24 weeks, and 70 to 85 percent after one year [25]. After an ulcer has healed, maintenance of compression therapy lifelong may reduce the risk of recurrence $[12,27,28]$. However, pain, drainage, application difficulty and physical limitations including obesity and contact dermatitis may be limiting factors to adhere to the therapy [22]. Contraindications to compression therapy include clinically significant arterial disease and uncompensated heart failure.

\subsection{Inelastic Compression Therapy}

It provides high working pressure during ambulation and muscle contraction, but no resting pressure. Unna boota zinc oxide impregnated, moist bandage that hardens after application, is the most common method of inelastic compression therapy which improves healing rates compared with placebo or hydroactive dressings [25,29]. However, a 2009 Cochrane review found that adding a component of elastic compression therapy is more effecttive than inelastic compression therapy alone [48]. Unna boot being inelastic, does not conform to changes in leg size and may be uncomfortable to wear. It may also cause accumulation of exudates from the ulcer leading to 
foul smell, requiring frequent reapplications [2].

\subsection{Elastic Compression Therapy}

Elastic compression therapy methods conform to changes in leg size and sustain compression during both rest and activity in contrast to Unna boot. Elastic compression can be achieved by stockings or bandages however elastic wraps (e.g., Ace wraps) do not provide enough pressure, and hence are not recommended for use [48]. Compression stockings are graded to exert the greatest pressure at the ankle and gradually decreasing pressure toward the knee and thigh. The pressure should be at least $20-30$ $\mathrm{mm} \mathrm{Hg}$ but preferably $30-44 \mathrm{~mm} \mathrm{Hg}$. Compression stockings are advised to be removed at night, and they should be changed to a new one, every six months as they lose pressure with regular washing [2]. Elastic bandages (e.g., Profore) are alternatives to compression stockings. Elastic compression therapy has been shown to be more effective than inelastic therapy in a recent meta-analysis [49]. In addition to that, high compression has been found to be more effective than low compression, and multilayer bandages are more effective than single layer $[26,48,50]$. The problem with multilayer compression bandages is that they are required to be applied by a skilled person preferably a physician, one or two times per week, depending on drainage.

\subsection{Intermittent Pneumatic Compression}

It comprises of a pump that delivers air to inflatable and deflatable sleeves that embrace extremities, providing intermittent compression $[7,26]$. The benefits when compared to standard continuous compression are not obvious and also it is expensive and requires immobilization of the patient; and therefore it is generally reserved for bedridden patients who cannot tolerate continuous compression therapy $[27,43]$.

\subsection{Leg Elevation}

Leg elevation along with compression therapy is also considered standard of care. It includes raising lower limbs above the level of the heart, with the aim of reducing edema, improving microcirculation and oxygen delivery and thereby hastening ulcer healing. In one small study, leg elevation increased the laser Doppler flux (i.e., flow within veins) by 45 percent [30]. Leg elevation is most effective if performed for 30 minutes, three or four times per day, which may be difficult for patients to follow in routine life.

\subsection{Dressings}

Dressings are done under compression bandages to promote faster healing and prevent adherence of the bandage to the ulcer. There are various dressings commercially available, including hydrocolloids (e.g., Duoderm), foams, hydrogels, pastes, and simple nonadherent dressings [14, 31]. A meta-analysis of 42 randomized controlled trials (RCTs) with a total of more than 1000 patients demonstrated no significant difference among many types of dressing [32]. Hence the choice of dressings for venous ulcers can be guided by cost, ease of application, and patient and physician preference [32].

\subsection{Mechanical Treatment}

Vacuum-assisted closure uses topical negative pressure and has shown to help reduce wound depth and volume compared with a hydrocolloid gel and gauze regimen for various wounds [33]. Though clinically meaningful outcomes, such as healing time has not yet been studied well and currently there is insufficient but high-quality data to support the use of topical negative pressure for venous ulcers [33].

\section{Medical Agents}

\subsection{Pentoxifylline}

Pentoxifylline is an inhibitor of platelet aggregation; it reduces blood viscosity and hence, improves microcirculation. At a dose $400 \mathrm{mg}$ three times per day, it has been shown to be an effective adjunct to compression therapy for the treatment for venous ulcers $[34,43]$. The patients who are unable to tolerate compression bandaging, pentoxifylline may also be useful as monotherapy [34]. The common adverse effects are gastrointestinal (e.g., nausea, vomiting, diarrhea, heartburn, loss of appetite). However, the cost-effectiveness of pentoxifylline has yet not been proved despite many studies to support its effectiveness as adjunctive therapy and monotherapy.

\subsection{Aspirin}

Adding aspirin therapy to compression bandages is recommended in the treatment of venous ulcers as long as there are no contraindications to its use and it has shown to increase ulcer healing time and reduce ulcer size, compared with compression therapy alone [35].

\subsection{Iloprost}

Iloprost is a vasodilator which inhibits platelet aggregation and when used with elastic compression therapy, it significantly reduces healing time of venous ulcers compared with placebo [36]. However, its high cost is a big issue and there are insufficient data to recommend its use [43].

\subsection{Oral Zinc}

Zinc has potential anti-inflammatory effects and has been 
shown to decrease healing time, however, oral zinc had not been found having any beneficial effect in the treatment of venous ulcers [37].

\subsection{Antibiotics/Antiseptics}

Secondary bacterial infection is a known problem to hamper the healing of the chronic wounds and venous ulcer as well. However routine use of systemic and topical antibiotics for the treatment of venous ulcers has not been recommended [1]. Presence or suspicion of cellulitis and osteomyelitis may warrant the use of antibiotics to treat the underlying infection.

\subsection{Hyperbaric Oxygen Therapy}

There is limited data to support the use of hyperbaric oxygen therapy as an adjunctive therapy for chronic wound healing because of potential anti-inflammatory and antibacterial effects, and its benefits in healing diabetic foot ulcers [38].

\subsection{Surgical Management}

The poor healing rates associated with chronic ulcers may raise the need for the surgical evaluation and management in patients with venous ulcers that are refractory to conservative therapies [51]. In general, after six months of treatment overall rate of healing in acute ulcers (duration of three months or less) is 71 to 80 percent whereas only 22 percent chance of healing in chronic ulcers [7].

\subsection{Debridement}

Debridement may be achieved by various means e.g. using sharps (e.g., using curette or scissors), enzymatic, mechanical, biologic or autolytic. The effect of debridement or no debridement and comparison of various types of debridement on the rate of venous ulcer healing has been studied variously [39-43]. In general, purely venous ulcers rarely need much debridement hence for the wounds with significant necrotic tissue, arterial insufficiency must be evaluated.

\subsection{Skin Grafting or Flap Coverage}

In order to give coverage to large or refractory venous ulcers; autograft, allograft or artificial skin may be used $[44,45,52]$. However, in presence of persistent edema and without treating the underlying venous disease, skin grafts may not be much effective [43]. Aggressive debridement of these ulcers may at times result in a large defect and may require coverage using flaps. A free flap transfer provides a local venous arborisation containing hundreds of competent valves and so it also hastens healing. The iliac crest with attached muscle can be used as a bone graft to take care of bone defects.

\subsection{Surgery for Venous Insufficiency}

The surgical intervention may help to reduce venous reflux, hasten healing, and prevent ulcer recurrence. Venous insufficiency may be taken care of surgically by saphenous vein ablation, subfascial endoscopic surgery for the interruption of the perforating veins; stenting for treatment of iliac vein obstruction and removal of incompetent superficial veins with phlebectomy, stripping, sclerotherapy, or laser therapy $[22,38,43]$. Surgical management has been shown to achieve an ulcer healing rate of 88 percent, with only a 13 percent recurrence rate over 10 months [46]. There should be an early evaluation for possible surgical intervention however the superiority of surgery over medical management has never been proved [47].

\subsection{Prophylaxis for Deep Vein Thrombosis}

Deep vein thrombosis is known to be an important cause of subsequent chronic venous insufficiency. Use of heaprin and pneumatic compression devices reduces the incidence of postoperative deep vein thrombosis. Outpatient surgery allows early mobilization and should be preferred if possible.

\subsection{Aftercare and Follow up}

Patients seen with early signs and symptoms of chronic venous insufficiency must have the cause ascertained. Correction of the causes of the chronic venous insufficiency has important role in preventing the development or recurrence of an ulcer. Patients should be instructed for using good quality foot wears, skin care, avoidance of prolonged standing, prevention of infections and trauma to the foot and the permanent use of compression stockings in order to prevent further deterioration, especially if surgical correction is not possible. A regular and vigilant follow up care by a trained person must be ensured after the ulcer has healed, and proper record should be maintained. To have good long term results continuous care, ulcer care clinics, home health nursing and regular evaluation by a doctor; are the pillars. They have a crucial role to play for the amelioration of this common and morbid condition.

\section{Conclusion}

Venous ulcers are fairly common problem in the present era. Though they are not common cause of mortality but they cause significant morbidity and impairment of work. Timely diagnosis and treatment may prevent the development of severe complication. Vigilant self care and 
regular follow up to a trained physician is the key of long term success.

\section{REFERENCES}

[1] S. O’Meara, D. Al-Kurdi and L. G. Ovington, “Antibiotics and Antiseptics for Venous Leg Ulcers," Cochrane Database Systematic Reviews, No. 1, 2008, Article ID: CD003557.

[2] T. de Araujo, I. Valencia, D. G. Federman and R. S. Kirsner, "Managing the Patient with Venous Ulcers," Annals of Internal Medicine, Vol. 138, No. 4, 2003, pp. 326-334.

[3] L. P. Abbade and S. Lastória, "Venous Ulcer: Epidemiology, Physiopathology, Diagnosis and Treatment," International Journal of Dermatology, Vol. 44, No. 6, 2005, pp. 449-456. doi:10.1111/j.1365-4632.2004.02456.x

[4] M. J. Callam, D. R. Harper, J. J. Dale and C. V. Ruckley, “Chronic Ulcer of the Leg: Clinical History," British Medical Journal, Vol. 294, No. 6584, 1987, pp. 1389-1391. doi:10.1136/bmj.294.6584.1389

[5] D. Bergqvist, C. Lindholm and O. Nelzén, "Chronic Leg Ulcers: The Impact of Venous Disease,” Journal of Vascular Surgery, Vol. 29, No. 4, 1999, pp. 752-755. doi:10.1016/S0741-5214(99)70330-7

[6] H. Ravaghi, K. Flemming, N. Cullum and A. Olyaee Manesh, "Electromagnetic Therapy for Treating Venous Leg Ulcers," Cochrane Database Systematic Reviews, No. 3, 2006, Article ID: CD002933.

[7] E. A. Nelson, S. E. Bell-Syer and N. A. Cullum, "Compression for Preventing Recurrence of Venous Ulcers," Cochrane Database Systematic Reviews, No. 4, 2000, Article ID: CD002303.

[8] M. Briggs and E. A. Nelson, "Topical Agents or Dressings for Pain in Venous Leg Ulcers," Cochrane Database Systematic Reviews, No. 1, 2003, Article ID: CD001177.

[9] O. Nelzén, D. Bergqvist and A. Lindhagen, "Long-Term Prognosis for Patients with Chronic Leg Ulcers: A Prospective Cohort Study," European Journal of Vascular \& Endovascular Surgery, Vol. 13, No. 5, 1997, pp. 500-508. doi:10.1016/S1078-5884(97)80179-7

[10] R. H. Samson and D. P. Showalter, "Stockings and the Prevention of Recurrent Venous Ulcers,” Dermatologic Surgery, Vol. 22, No. 4, pp. 373-376. doi:10.1016/1076-0512(95)00030-5

[11] M. J. Callam, C. V. Ruckley, D. R. Harper and J. J. Dale, "Chronic Ulceration of the Leg: Extent of the Problem and Provision of Care,” British Medical Journal, Vol. 290, No. 6485, 1985, pp. 1855-1856. doi:10.1136/bmj.290.6485.1855

[12] C. V. Ruckley, "Socioeconomic Impact of Chronic Venous Insufficiency and Leg Ulcers,” Angiology, Vol. 48, No. 1, 1997, pp. 67-69. doi:10.1177/000331979704800111

[13] I. C. Valencia, A. Falabella, R. S. Kirsner and W. H. Eaglstein, "Chronic Venous Insufficiency and Venous Leg Ulceration," Journal of the American Academy of Dermatology, Vol. 44, No. 3, 2001, pp. 401-421. doi:10.1067/mjd.2001.111633
[14] C. N. Etufugh and T. J. Phillips, "Venous Ulcers,” Clinics in Dermatology, Vol. 25, No. 1, 2007, pp. 121-130. doi:10.1016/j.clindermatol.2006.09.004

[15] J. Grey, K. Harding and S. Enoch, "ABC of Wound Healing: Venous and Arterial Leg Ulcers,” British Medical Journal, Vol. 332, 2006, p. 7537. doi:10.1136/bmj.332.7537.347

[16] H. Newton and J. Cameron, "Skin Care in Wound Management," Medical Communications UK Ltd., Holsworthy, 2003.

[17] I. Anderson, "What Is a Venous Leg Ulcer?” Wound Essentials, Vol. 4, 2009, pp. 36-44.

[18] T. J. Phillips and J. S. Dover, "Leg Ulcers," Journal of the American Academy of Dermatology, Vol. 25, No. 6, 1991, pp. 965-987. doi:10.1016/0190-9622(91)70295-D

[19] S. R. McGee and E. J. Boyko, "Physical Examination and Chronic Lower-Extremity Ischemia: A Critical Review,” Archives of Internal Medicine, Vol. 158, No. 12, 1998, pp. 1357-1364. doi:10.1001/archinte.158.12.1357

[20] J. M. Scriven, T. Hartshorne, P. R. Bell, A. R. Naylor and N. J. London, "Single-Visit Venous Ulcer Assessment Clinic: The First Year,” British Journal of Surgery, Vol. 84, No. 3, 1997, pp. 334-336. doi:10.1002/bjs.1800840319

[21] A. Lopez and T. Phillips, "Venous Ulcers," Wounds, Vol. 10, No. 5, 1998, pp. 149-157.

[22] S. Raju and P. Neglén, "Clinical Practice. Chronic Venous Insufficiency and Varicose Veins,” The New England Journal of Medicine, Vol. 360, No. 22, 2009, pp. 23192327. doi:10.1056/NEJMcp0802444

[23] B. Eklöf, R. B. Rutherford, J. J. Bergan, et al., "Revision of the CEAP Classification for Chronic Venous Disorders: Consensus Statement," Journal of Vascular Surgery, Vol. 40, No. 6, 2004, pp. 1248-1252. doi:10.1016/j.jvs.2004.09.027

[24] A. J. Stewart and D. J. Leaper, "Treatment of Chronic Ulcers in the Community: A Comparison of Scherinsorb and Iodosorb,” Phlebology, Vol. 2, 1987, pp. 115-121.

[25] D. J. Margolis, J. A. Berlin and B. L. Strom, "Which Venous Leg Ulcers Will Heal with Limb Compression Bandages?” The American Journal of Medicine, Vol. 109, No. 1, 2000, pp. 15-19. doi:10.1016/S0002-9343(00)00379-X

[26] A. Fletcher, N. Cullum and T. A. Sheldon, "A Systematic Review of Compression Treatment for Venous Leg Ulcers,” British Medical Journal, Vol. 315, No. 7108, 1997, pp. 576-580. doi:10.1136/bmj.315.7108.576

[27] T. J. Phillips, F. Machado, R. Trout, J. Porter, J. Olin and V. Falanga, "Prognostic Indicators in Venous Ulcers," Journal of the American Academy of Dermatology, Vol. 43, No. 4, 2000, pp. 627-630. doi:10.1067/mjd.2000.107496

[28] E. A. Nelson, R. Mani and K. Vowden, "Intermittent Pneumatic Compression for Treating Venous Leg Ulcers," Cochrane Database Systematic Reviews, No. 2, 2008, Article ID: CD001899.

[29] M. J. Kikta, J. J. Schuler, J. P. Meyer, et al., “A Prospective, Randomized Trial of Unna's Boots versus Hydroactive Dressing in the Treatment of Venous Stasis Ulcers," Journal of Vascular Surgery, Vol. 7, No. 3, 1988, pp. 478- 
483.

[30] A. Abu-Own, J. H. Scurr and P. D. Coleridge Smith, "Effect of Leg Elevation on the Skin Microcirculation in Chronic Venous Insufficiency,” Journal of Vascular Surgery, Vol. 20, No. 5, 1994, pp. 705-710. doi:10.1016/S0741-5214(94)70157-1

[31] S. Seaman, "Dressing Selection in Chronic Wound Management," Journal of the American Podiatric Medical Association, Vol. 92, No. 1, 2002, pp. 24-33.

[32] S. Palfreyman, E. A. Nelson and J. A. Michaels, "Dressings for Venous Leg Ulcers: Systematic Review and MetaAnalysis,” British Medical Journal, Vol. 335, No. 7613, 2007, p. 244. doi:10.1136/bmj.39248.634977.AE

[33] D. T. Ubbink, S. J. Westerbos, D. Evans, L. Land and H. Vermeulen, "Topical Negative Pressure for Treating Chronic Wounds," Cochrane Database Systematic Reviews, No. 3, 2008, Article ID: CD001898.

[34] A. Jull, J. Waters and B. Arroll, "Pentoxifylline for Treatment of Venous Leg Ulcers: A Systematic Review," Lancet, Vol. 359, No. 9317, 2002, pp. 1550-1554. doi:10.1016/S0140-6736(02)08513-6

[35] A. M. Layton, S. H. Ibbotson, J. A. Davies and M. J. Goodfield, "Randomised Trial of Oral Aspirin for Chronic Venous Leg Ulcers,” Lancet, Vol. 344, No. 8916, 1994, pp. 164-165. doi:10.1016/S0140-6736(94)92759-6

[36] F. Ferrara, F. Meli, F. Raimondi, et al., "The Treatment of Venous Leg Ulcers: A New Therapeutic Use of Iloprost," Annals of Surgery, Vol. 246, No. 5, 2007, pp. 860865. doi:10.1097/SLA.0b013e3180caa44c

[37] E. A. Wilkinson and C. I. Hawke, "Does Oral Zinc Aid the Healing of Chronic Leg Ulcers? A Systematic Literature Review,” Archives of Dermatology, Vol. 134, No. 12, 1998, pp. 1556-1560. doi:10.1001/archderm.134.12.1556

[38] P. Kranke, M. Bennett, I. Roeckl-Wiedmann and S. Debus, "Hyperbaric Oxygen Therapy for Chronic Wounds," Cochrane Database Systematic Reviews, No. 2, 2004, Article ID: CD004123.

[39] C. Lok, C. Paul, P. Amblard, et al., "EMLA Cream as a Topical Anesthetic for the Repeated Mechanical Debridement of Venous Leg Ulcers: A Double-Blind, Placebo-Controlled Study," Journal of the American Academy Dermatology, Vol. 40, No. 2, 1999, pp. 208-213. doi:10.1016/S0190-9622(99)70190-8

[40] A. F. Falabella, P. Carson, W. H. Eaglstein and V. Falanga, "The Safety and Efficacy of a Proteolytic Ointment in the Treatment of Chronic Ulcers of the Lower Extremity," Journal of the American Academy Dermatology, Vol. 39, No. 5, 1998, pp. 737-740. doi:10.1016/S0190-9622(98)70045-3

[41] J. C. Dumville, G. Worthy, J. M. Bland, et al., "Larval Therapy for Leg Ulcers (VenUS II): Randomized Controlled Trial,” British Medical Journal, Vol. 338, 2009, p. b773. doi:10.1136/bmj.b773
[42] M. O. Soares, C. P. Iglesias, J. M. Bland, et al., "Cost Effectiveness Analysis of Larval Therapy for Leg Ulcers," British Medical Journal, Vol. 338, 2009, p. b825. doi:10.1136/bmj.b825

[43] M. C. Robson, D. M. Cooper, R. Aslam, et al., "Guidelines for the Treatment of Venous Ulcers," Wound Repair and Regeneration, Vol. 14, No. 6, 2006, pp. 649-662. doi:10.1111/j.1524-475X.2006.00174.X

[44] J. E. Jones and E. A. Nelson, "Skin Grafting for Venous Leg Ulcers," Cochrane Database Systematic Reviews, No. 2, 2007, Article ID: CD001737.

[45] V. Falanga, D. Margolis, O. Alvarez, et al., "Rapid Healing of Venous Ulcers and Lack of Clinical Rejection with an Allogeneic Cultured Human Skin Equivalent. Human Skin Equivalent Investigators Group,” Archives of Dermatology, Vol. 134, No. 3, 1998, pp. 293-300. doi:10.1001/archderm.134.3.293

[46] J. R. Barwell, C. E. Davies, J. Deacon, et al., "Comparison of Surgery and Compression with Compression Alone in Chronic Venous Ulceration (ESCHAR Study): Randomized Controlled Trial,” Lancet, Vol. 363, No. 9424, 2004, pp. 1854-1859. doi:10.1016/S0140-6736(04)16353-8

[47] J. A. Tenbrook Jr., M. D. Iafrati, T. F. O’donnell Jr., et al., "Systematic Review of Outcomes after Surgical Management of Venous Disease Incorporating Subfascial Endoscopic Perforator Surgery," Journal of Vascular Surgery, Vol. 39, No. 3, 2004, pp. 583-589. doi:10.1016/j.jvs.2003.09.017

[48] S. O’Meara, N. A. Cullum and E. A. Nelson, "Compression for Venous Leg Ulcers," Cochrane Database Systematic Reviews, No. 1, 2009, Article ID: CD000265.

[49] S. O’Meara, J. Tierney, N. Cullum, et al., "Four Layer Bandage Compared with Short Stretch Bandage for Venous Leg Ulcers: Systematic Review and Meta-Analysis of Randomised Controlled Trials with Data from Individual Patients,” British Medical Journal, Vol. 338, 2009, p. b1344. doi:10.1136/bmj.b1344

[50] N. Cullum, E. A. Nelson, A. W. Fletcher and T. A. Sheldon, "Compression Bandages and Stockings for Venous Ulcers," Cochrane Database Systematic Reviews, No. 2, 2000, Article ID: CD000265.

[51] P. Tallman, E. Muscare, P. Carson, W. H. Eaglstein and V. Falanga, "Initial Rate of Healing Predicts Complete Healing of Venous Ulcers," Archives of Dermatology, Vol. 133, No. 10, 1997, pp. 1231-1234. doi:10.1001/archderm.1997.03890460053006

[52] W. S. Douglas and N. B. Simpson, "Guidelines for the Management of Chronic Venous Leg Ulceration. Report of a Multidisciplinary Workshop. British Association of Dermatologists and the Research Unit of the Royal College of Physicians,” British Journal of Dermatology, Vol. 132, No. 3, 1995, pp. 446-452. doi:10.1111/j.1365-2133.1995.tb08681.x 